\title{
Design and Analytical Analysis of Foundation Pile Ground Heat Exchanger with Spiral Coils
}

\section{Yi Man}

\author{
Yunxia Qu
}

\author{
Zejiang Wang
}

Zhaohong Fang

\begin{abstract}
Recently, utilization of building foundation piles as the ground beat exchanger (GHE) received more and more attention since it can reduce the initial cost and land area requirement compared with the borehole GHE. This study designs a foundation pile GHE with spiral coil (FPGHE) by intertwing the circulating coil pipe tightly in spiral shape against the reinforcing steel of a pile. The distinct advantage of this proposed FPGHE is that it can offer bigher heat transfer efficiency, reduce pipe connection complexity, prevent air blocking and decrease the thermal "short-circuit" between the feed and return pipes compared with other existing configurations. In order to analyze its heat transfer characteristic, analytical models are established for the proposed FPGHE. Analytical thermal analysis is carried out to simulate temperature responses of the coil pipe wall and the circulating water entering/effusing the FPGHE to the short time step heat transfer loads based on the established analytical model. Furthermore, the operation performance and heat exchange capacity of the FPGHE is investigated.
\end{abstract}

\section{INTRODUCTION}

The ground heat exchangers (GHE) with vertical boreholes (Bose, et al. 1985) have been the mainstream technology for the ground coupled heat pump systems, but the high initial cost and land area requirement to install the borehole GHE remain the major obstacles of this technology. Since the foundation pile is commonly used in high rise buildings, combining the heat exchanger and building foundation pile can eliminate the drilling expense and land area requirement of borehole GHE. Therefore, utilization of building foundation piles as the GHE has received more and more attention(Mehrizi, et al. 2016; Luo, et al. 2016; Huerta and Krarti 2015; Loveridge and Powrie 2014). In existing studies, pipes are usually buried in foundation piles in configurations of U-tubes, W-tubes or spiral coils.

For the first two configurations, the heat transfer area inside foundation pile is small and the air blocking may occur in pipes. In order to overcome these drawbacks, this study focus on the third type and designs a foundation pile GHE with spiral coil (FPGHE). The circulation coil pipe is intertwined tightly in spiral shape against the reinforcing steel of a pile, and is disposed within about $0.1 \mathrm{~m}$ of the pile's outer surface. The distinct advantage of FPGHE is that it can offer higher heat transfer efficiency, reduce pipe connection complexity, prevent air blocking and decrease the thermal "short-circuit" between the feed and return pipes compared with other existing configurations. The schematic diagrams of a conventional single U-tube vertical borehole GHE and the FPGHE with spiral coils are compared in Figure 1. 

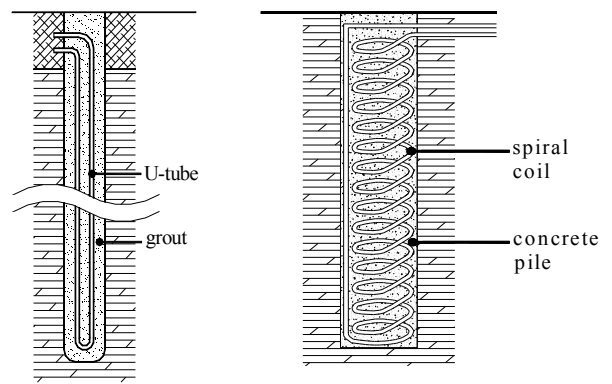

Figure 1 Schematic diagram of a vertical borehole and a FPGHE with spiral coil

Modeling the FPGHE with spiral coils is complex and existing studies concentrated on the numerical or experimental methods. Suryatriyastuti carried out a 3-D numerical heat transfer analysis on the energy pile under the constant heat load case (Suryatriyastuti, et al. 2012). Xiang built a numerical model includes a 1-D transient convection-diffusion submodel for the fluid domain and a 1-D transient diffusion submodel for the solid domain (Xiang, et al. 2015). Luo conducted the thermal performance test to analyze the operation performance of energy pile under an intermittent condition (Luo, et al. 2016). In the present study, an analytical method is explored as it can provide a more practical and convenient tool for engineering design, as well as thermal analysis of the FPGHE, compared with existing numerical and experimental methods.

For the proposed FPGHE, its diameter is much thicker and depth is usually shorter compared with the borehole GHE. Obviously, classical heat transfer models for the borehole GHE fail for the FPGHE. By analyzing the heat transfer process of proposed FPGHE, the analytical finite spiral heat source model is established in this study based on the Green's function theory, the virtual heat source theory, and the superposition method. The temperature responses of the spiral heat source, the coil pipe wall, and the circulating water entering/effusing the FPGHE to the short time step heat transfer loads are deduced based on the established analytical model. Then the operation performance and the heat exchange capacity of the FPGHE is investigated.

\section{DESIGN OF FOUNDATION PILE GHE WITH SPIRAL COIL}

The high-density polyethylene (HDPE) pipe with exterior and interior diameters of $25 \mathrm{~mm}$ and $20 \mathrm{~mm}$, respectively, are selected as the circulation pipe of proposed FPGHE. First hydrostatic test with pressure of 0.8Mpa and last for $15 \mathrm{~min}$ are required in order to prevent the leakage before the pipe are installed. Then the pipe is intertwined tightly in spiral shape against the reinforcing steel cage of a pile, as shown in Figure 2(a). The second hydrostatic test with pressure of $0.8 \mathrm{Mpa}$ and last for $15 \mathrm{~min}$ should be carried out followed. Then the combination of reinforcing steel cage and coil pipe are put into the hole of pile. After the third hydrostatic test with pressure of $0.8 \mathrm{Mpa}$ and last for 2 hours, the last step is the concrete pouring of the pile foundation, as shown in Figure 2(b).

Extreme caution should be paid during the concrete pouring, and coarse aggregate in concrete must be smooth and non-angular particles. The conduit are utilized to lead the concrete into the bottom of pile hole, and it should be extracted gradually from the bottom to top according to grouting speed. During the placement and extraction process of conduit, it is important to keep the vertical and center for preventing the hanging cage of concrete, ensuring the compaction of pouring and decreasing the heat transfer resistance. The concrete pouring process is finished when the density of return slurry is identical with which of pouring concrete. 


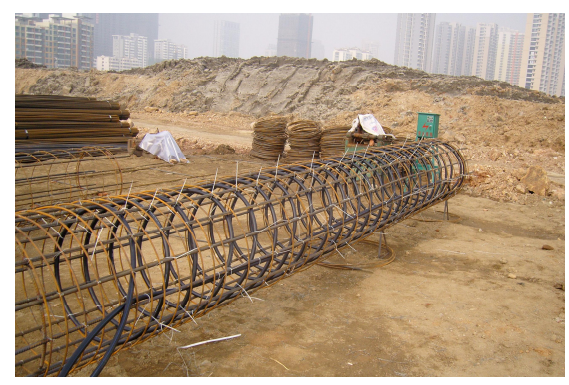

(a)

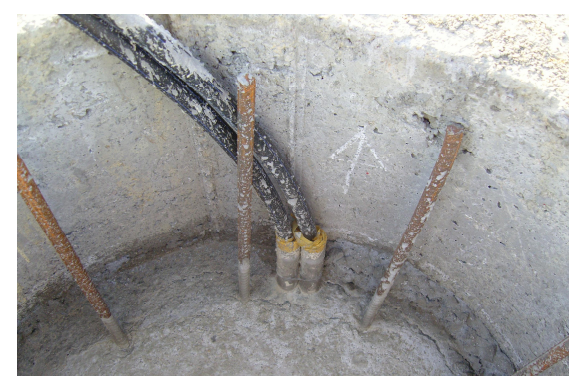

(b)

Figure 2 Circulation pipe intertwined with steel cage and concrete pouring of the pile foundation

\section{SPIRAL HEAT SOURCE MODEL ESTABLISHMENT}

As shown in Figure 3, the buried coil inside FPGHE is simplified into heat source in spiral shape with finitelength. Then the finite spiral heat source model is developed to take the three-dimensional geometrical characteristic of the spiral pile as well as the effects of heat flow through the top and bottom ends of pile into account.
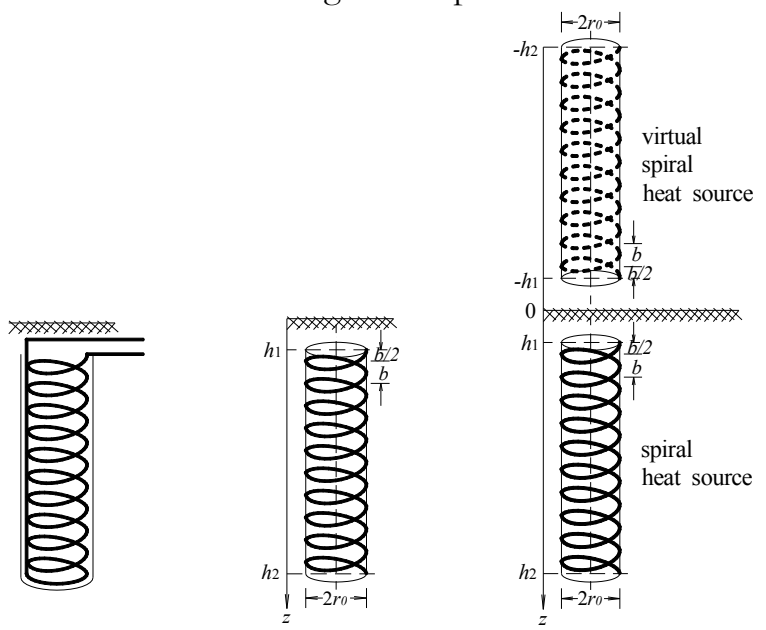

Figure 3 Proposed FPGHE, the finite spiral heat source model and the virtual heat source model

\section{Green's Function and Temperature Response to a Point Heat Source}

According to the Green's function theory, the Green's function is the temperature response to an instantaneous point source of heat. For an instantaneous point heat source with intensity of $\rho c$, located at $\left(r^{\prime}, \varphi^{\prime}, z^{\prime}\right)$ and activated at the instant $\tau^{\prime}$, its Green's function in the cylindrical coordinates at point $(r, \varphi, z)$ can be expressed as:

$$
G\left(r, \varphi, z, \tau ; r^{\prime}, \varphi^{\prime}, z^{\prime}, \tau^{\prime}\right)=\frac{1}{8\left[\pi a\left(\tau-\tau^{\prime}\right)\right]^{3 / 2}} \cdot \exp \left[-\frac{\left(r \cos \varphi-r^{\prime} \cos \varphi^{\prime}\right)^{2}+\left(r \sin \varphi-r^{\prime} \sin \varphi^{\prime}\right)^{2}+\left(z-z^{\prime}\right)^{2}}{4 a\left(\tau-\tau^{\prime}\right)}\right]
$$

Then the temperature response in the medium resulted from the step heating of the spiral heat source can be expressed as the integration of all the point sources acted successively from the starting instant $\tau^{\prime}=0$.

\section{Temperature Response to the Finite Spiral Heat Source}


As in most analytical models, assumptions are made for finite spiral source model as follows:

(1) The ground is regarded as a homogeneous semi-infinite medium, and its thermophysical properties do not change with temperature.

(2) The boundary of the medium, i.e. the ground surface, remains constant throughout the period concerned.

(3) The medium has a uniform initial temperature, $t_{0}$.

(4) The spiral heat source with radius $r_{0}$ and pitch $b$ stretches from the $b_{1}$ to $b_{2}$ below the ground surface, and each circle of the spiral sources emits constant heat transfer rate at the intensity of $q b$ from time zero, i.e. $\tau=0$.

With the virtual heat source theory, a virtual spiral heat sink with negative heating rate $-q_{l}$ and of identical physical dimensions is set on symmetry to the boundary in order to keep the constant temperature of the ground surface, as shown in Figure 3. The finite spiral heat source and heat sink can then be approximated as the sum of numerous point heat sources and heat sinks. Then, the Green's function theory and the superposition method is employed to obtain the temperature response of the medium:

$$
\begin{aligned}
\theta_{f, \text { spiral }} & =\frac{q_{l} b}{2 \pi \rho c} \int_{0}^{\tau} d \tau^{\prime}\left[\int_{2 \pi h / b}^{2 \pi h / b} G\left(z^{\prime}=b \varphi^{\prime} / 2 \pi\right) d \varphi^{\prime}-\int_{2 \pi h / / b}^{2 \pi h / b} G\left(z^{\prime}=-b \varphi^{\prime} / 2 \pi\right) d \varphi^{\prime} d \varphi^{\prime}\right] \\
& =\frac{q_{l} b}{16 \pi \rho c} \int_{0}^{\tau} \frac{d \tau^{\prime}}{\left[\pi a\left(\tau-\tau^{\prime}\right)\right]^{3 / 2}} \cdot \exp \left[-\frac{r^{2}+r_{0}^{2}}{4 a\left(\tau-\tau^{\prime}\right)}\right] . \\
& \int_{2 \pi h / b}^{2 \pi h / b} \exp \left[\frac{2 r r_{0} \cos \left(\varphi-\varphi^{\prime}\right)}{4 a\left(\tau-\tau^{\prime}\right)}\right]\left\{\exp \left[-\frac{\left(z-b \varphi^{\prime} / 2 \pi\right)^{2}}{4 a\left(\tau-\tau^{\prime}\right)}\right]-\exp \left[-\frac{\left(z+b \varphi^{\prime} / 2 \pi\right)^{2}}{4 a\left(\tau-\tau^{\prime}\right)}\right]\right\} d \varphi^{\prime}
\end{aligned}
$$

By introducing the following dimensionless variables: $\Theta_{\text {spiral }}=\frac{k \theta_{\text {spiral }}}{q_{l}}, F o=\frac{a \tau}{r_{0}^{2}}, F o^{\prime}=\frac{a \tau^{\prime}}{r_{0}^{2}}, R=\frac{r}{r_{0}}, Z=\frac{z}{r_{0}}, Z^{\prime}=\frac{z^{\prime}}{r_{0}}, B=\frac{b}{r_{0}}$, $H_{1}=\frac{h_{1}}{r_{0}}$ and $H_{2}=\frac{h_{2}}{r_{0}}$, the dimensionless temperature excess around the finite spiral source can be expressed as:

$$
\begin{aligned}
& \Theta_{f, \text { spiral }}=\frac{B}{16 \pi^{5 / 2}} \int_{0}^{F_{o}}\left(\frac{1}{F o-F o^{\prime}}\right)^{3 / 2} \cdot \exp \left[-\frac{R^{2}+1}{4\left(F o-F o^{\prime}\right)}\right] . \\
& \int_{2 \pi H_{1} / B}^{2 \pi H_{2} / B} \exp \left[\frac{2 R \cos \left(\varphi-\varphi^{\prime}\right)}{4\left(F o-F o^{\prime}\right)}\right] \cdot\left\{\exp \left[-\frac{\left(Z-B \varphi^{\prime} / 2 \pi\right)^{2}}{4\left(F o-F o^{\prime}\right)}\right]-\exp \left[-\frac{\left(Z+B \varphi^{\prime} / 2 \pi\right)^{2}}{4\left(F o-F o^{\prime}\right)}\right]\right\} d \varphi^{\prime} d F o^{\prime}
\end{aligned}
$$

\section{ANALYTICAL SOLUTIONS AND DISCUSSION}

\section{Temperature Field Solutions of Finite Spiral Source Model}

The temperature responses of the finite spiral source model can be calculated according to Equation (3). Take an example of finite spiral source with $B=1, H_{1}=2.0$, and $H_{2}=12.0$, the temperature fields covering the pile and its surrounding soil at different dimensionless times of $F_{0}=0.2, F_{0}=1.0$ and $F_{0}=5.0$ are described in Figure 4. As shown, the temperature rise fluctuates considerably in the vicinity to the spiral source and shows a periodic variation in the $Z$ direction. Besides, the influence of the axial heat conduction caused by the boundary and finite length of the heat source is limited to the two ends of the pile in relatively short periods of time, and the impact of heat dissipation through the ends penetrate deeper into the whole depth of the pile for longer term operation. 

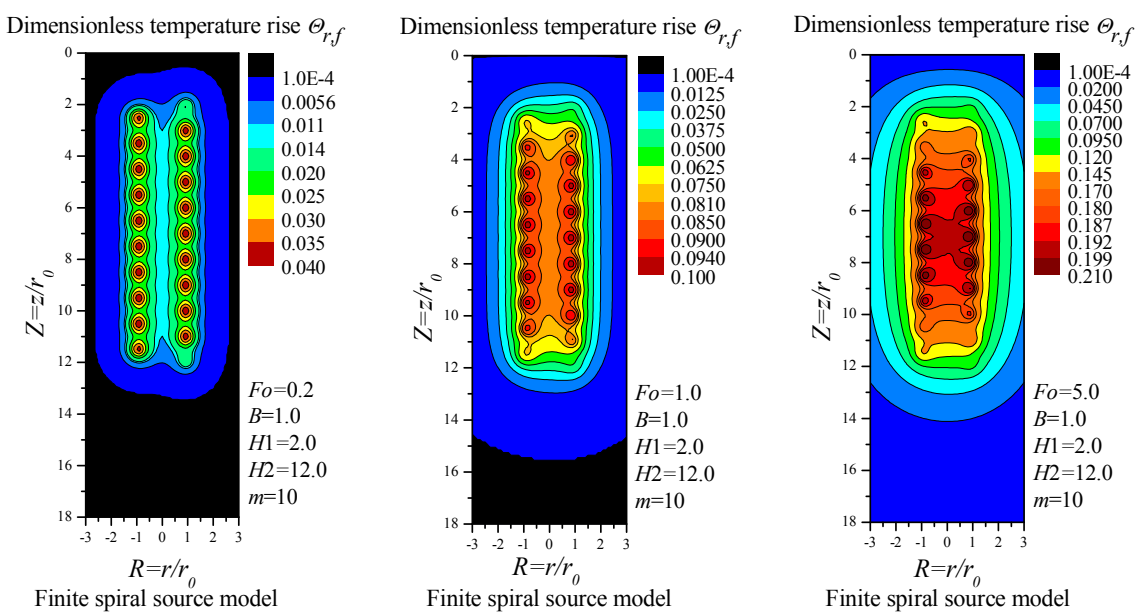

Figure 4 Temperature response of finite spiral source model

\section{Temperature Response of Pipe Wall}

When the dimension of the coil pipe is considered, the heat source can be approximated as located at the centre of coil pipe and the temperature response of the coil pipe wall located at $r_{p}$ away from spiral heat source can be deduced. Points on the spiral heat source can be located by the coordinate $\varphi$ with the other coordinates being $r=r_{0}$ and $z=z_{0}$ in the cylindrical coordinates, as shown in Figure 5.

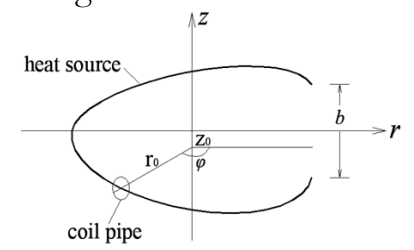

Figure 5 Spiral coil pipe buried in pile GHE

Consequently, while the slope of the spiral is neglected, the coordinates of a point on the pipe outer circumference can be determined approximately as:

$$
\left\{\begin{array}{l}
r=r_{0}+r_{p} \cdot \cos \alpha \\
\varphi=\varphi \\
z=z_{0}+r_{p} \cdot \sin \alpha \quad 0<\alpha<2 \pi
\end{array}\right.
$$

By introducing the dimensionless variables $R=\frac{r}{r_{0}}, Z=\frac{z}{r_{0}}, R_{p}=\frac{r_{p}}{r_{0}}, Z_{0}=\frac{z_{0}}{r_{0}}, B=\frac{b}{r_{0}}$, the dimensionless coordinate values of points located on the coil pipe wall becomes:

$$
\left\{\begin{array}{l}
R=\frac{r}{r_{0}}=\frac{r_{p}}{r_{0}} \cdot \cos \alpha+1=R_{p} \cdot \cos \alpha+1 \\
Z=\frac{z}{r_{0}}=\frac{r_{p}}{r_{0}} \cdot \sin \alpha+Z_{0}=R_{p} \cdot \sin \alpha+\frac{B \varphi}{2 \pi} \quad 0<\alpha<2 \pi
\end{array}\right.
$$

Then the temperature distribution on the coil pipe wall can be deduced according to the Equation (3): 


$$
\begin{aligned}
& \Theta_{f, \text { spiral pipe }}(\alpha, \varphi, F o)=\frac{B}{16 \pi^{5 / 2}} \int_{0}^{F_{0}}\left(\frac{1}{F o-F o^{\prime}}\right)^{3 / 2} \cdot \exp \left[-\frac{\left(R_{p} \cdot \cos \alpha+1\right)^{2}+1}{4\left(F o-F O^{\prime}\right)}\right] . \\
& \int_{2 \pi H_{1} / B}^{2} \exp \left[\frac{2\left(R_{p} \cdot \cos \alpha+1\right) \cos \left(\varphi-\varphi^{\prime}\right)}{4\left(F o-F O^{\prime}\right)}\right] \cdot \\
& \left\{\exp \left[-\frac{\left(R_{p} \cdot \sin \alpha+B / 2 \pi \cdot\left(\varphi-\varphi^{\prime}\right)\right)^{2}}{4\left(F o-F o^{\prime}\right)}\right]-\exp \left[-\frac{\left(R_{p} \cdot \sin \alpha+B / 2 \pi \cdot\left(\varphi+\varphi^{\prime}\right)\right)^{2}}{4\left(F o-F O^{\prime}\right)}\right]\right\} d \varphi^{\prime} d F o^{\prime}
\end{aligned}
$$

For engineering application, the integral mean temperature response of the coil pipes wall over its circumference in z-direction can be selected to represent the pipe wall temperature response of the whole FPGHE.

\section{Coil Pipe Wall Temperature Response to the Practical Heat Current}

The practical mutative heat currents imposed on the FPGHE can be approximated by the sum of a serious of rectangular pulse heat currents. Further, a single pulse heat current can be approximated by superposition of two step heat currents with the initial one $q_{l_{0}}=0$. Therefore, concepts of $p$-function and $q$-function which represent the nondimensional temperature response of the pipe wall to the step heat current and the pulse heat current, respectively, are developed to calculate the pipe wall temperature response to practical heat current which varies from time to time:

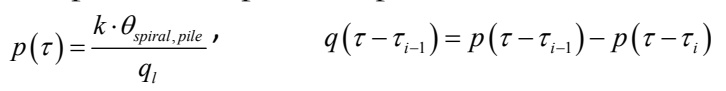

According to superimposing theory, the temperature response of the borehole wall at moment $\tau$ can be deduced based on the short time step pulse heat currents $q_{l_{i}}$ :

$$
\begin{aligned}
& \theta_{\text {spiral }, \text { pile }}=\frac{1}{k} \sum_{i=1}^{\infty}\left(q_{l_{i}}-q_{l_{l-1}}\right) \cdot p\left(\tau-\tau_{i-1}\right) \\
& =\frac{1}{k}\left[\sum_{i=1}^{\infty} q_{l_{i}} \cdot p\left(\tau-\tau_{i-1}\right)-\sum_{i=1}^{\infty} q_{l_{i-1}} \cdot p\left(\tau-\tau_{i-1}\right)\right]=\frac{1}{k}\left[\sum_{i=1}^{\infty} q_{l_{i}} \cdot p\left(\tau-\tau_{i-1}\right)-q_{l_{0}} \cdot p\left(\tau-\tau_{0}\right)-\sum_{j=1}^{\infty} q_{l^{\prime}} \cdot p\left(\tau-\tau_{j}\right)\right] \\
& =\frac{1}{k} \sum_{i=1}^{\infty} q_{l_{i}} \cdot\left[p\left(\tau-\tau_{i-1}\right)-p\left(\tau-\tau_{i}\right)\right]=\frac{1}{k} \sum_{i=1}^{\infty} q_{l_{i}} q\left(\tau-\tau_{i-1}\right)
\end{aligned}
$$

\section{Temperature of Circulating Fluid}

The temperature of fluid circulating in the coil pipe is of primary importance to design and simulate the FPGHE. Compared with the ground outside the pile, both the dimensional scale and thermal mass of the coil pipe are much smaller. Moreover, the temperature variation inside the coil pipe is usually slow and minor. Thus, the heat transfer of fluid inside the FPGHE can be approximated as a steady-state process. The heat transfer resistance between the coil pipe external wall and circulating fluid can be calculated:

$$
R_{r p}=\frac{1}{2 \pi k_{p}} \ln \left(\frac{r_{p}}{r_{p i}}\right)+\frac{1}{2 \pi r_{p i} h_{f}}
$$

where $h_{f}$ is the convection heat transfer coefficient between the pipe wall and circulating fluid.

$$
h_{f}=\varepsilon_{R} \cdot \frac{k_{f}}{2 r_{p i}} N u
$$

$\mathrm{Nu}$ is the Nusselt number for a straight pipe. $\varepsilon_{R}$ is an amendatory factor (Rohsenow and Hartnett 1983) of the convection heat transfer coefficient due to the augment effect of centrifugal force of fluid flow in the spiral channel.

$$
\varepsilon_{R}=1+10.3\left(\frac{2 r_{p}}{r_{0}}\right)^{3}
$$

Due to the steady-state process simplification, the average temperature of circulating fluid can be calculated:

$$
T_{f}=\frac{T_{f}{ }^{\prime}+T_{f}{ }^{\prime \prime}}{2}=T_{p}+\frac{q_{l} \cdot b}{\sqrt{\left(2 \pi \cdot r_{0}\right)^{2}+b^{2}}} \cdot R_{i p}
$$


In view of heat balance for the FPGHE, one also has:

$$
\Delta T=T_{f}{ }^{\prime}-T_{f}{ }^{\prime \prime}=\frac{q_{l}\left(h_{2}-h_{1}\right)}{M \cdot C_{p}}
$$

Then the entering and effusing fluid temperatures of the FPGHE can be determined by:

$$
\left\{\begin{array}{l}
T_{f}{ }^{\prime}=T_{p}+\frac{q_{l} \cdot b \cdot R_{r p}}{\sqrt{\left(2 \pi \cdot r_{0}\right)^{2}+b^{2}}}+\frac{q_{l}\left(h_{2}-h_{1}\right)}{2 M \cdot C_{p}} \\
T_{f}{ }^{\prime \prime}=T_{p}+\frac{q_{l} \cdot b \cdot R_{r p}}{\sqrt{\left(2 \pi \cdot r_{0}\right)^{2}+b^{2}}}-\frac{q_{l}\left(h_{2}-h_{1}\right)}{2 M \cdot C_{p}}
\end{array}\right.
$$

\section{Operation Simulation of a Sample FPGHE}

A FPGHE with normal pile configurations for engineering application is selected as a sample in this study: $r_{0}=0.4 \mathrm{~m}, h_{1}=2 \mathrm{~m}, h_{2}=22 \mathrm{~m}, b=0.4 \mathrm{~m}, r_{p i}=20 \mathrm{~mm}$ and $r_{p}=32 \mathrm{~mm}$. The flow velocity of fluid inside coil pipe is $0.5 \mathrm{~m} / \mathrm{s}$ for ensuring the turbulent flow. The undisturbed temperature of surrounding soil is $12.5{ }^{\circ} \mathrm{C}$. The hourly heat extraction/injection loads afforded by the sample FPGHE is assigned by an air conditioning loads software simulation program, as shown in Figure 6(a). Then the hourly operation parameters of the sample FPGHE in one year are simulated according to the proposed analytical model and plotted in Figure 6(b). According to simulative results, the maximum and minimum temperature effusing the FPGHE for cooling and heating provision is $28.42^{\circ} \mathrm{C}$ and $3.81^{\circ} \mathrm{C}$, respectively. The heat exchange capacity of the sample FPGHE is about $212 \mathrm{~W} / \mathrm{m}$.

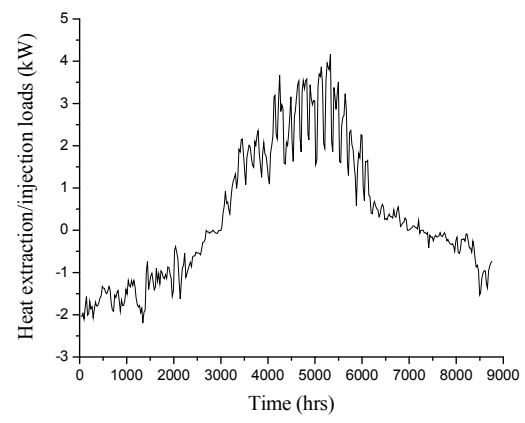

(a)

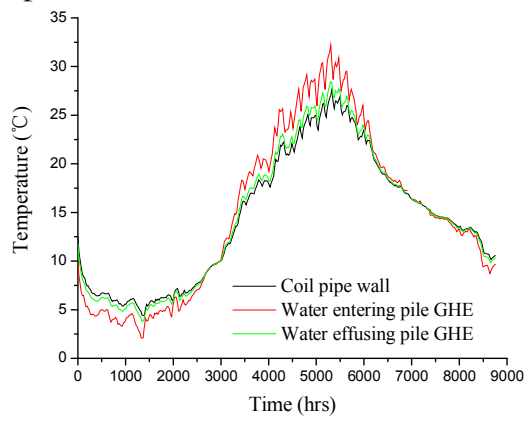

(b)

Figure 6 Heat change loads and simulative hourly operation parameters of sample FPGHE

\section{CONCLUSION}

This paper presents a model of a FPGHE with spiral coil, which can offer higher heat transfer efficiency, reduce pipe connection complexity, prevent air blocking and decrease the thermal "short-circuit" between the feed and return pipes. The design and manufacture key points of proposed FPGHE are illustrated.

In order to provide an appropriate and convenient tool for design and thermal analysis of the FPGHE with spiral coils, the analytical finite spiral heat source model is established in this study based on the Green's function theory, the virtual heat source theory, and the superposition method. The temperature responses of the spiral heat source, the coil pipe wall, and the circulating water entering/effusing the FPGHE to the short time step heat transfer loads are deduced based on the established analytical model. Then the operation performance and the heat exchange capacity of the FPGHE is investigated. Analytical analysis developed in this study can provide an proper method for design and thermal analysis of the FPGHE with spiral coils as well as other similar engineering problems. For further research, the optimal design parameters, such as coil pitch, of proposed FPGHE should be discussed based on the established spiral heat source model and other relevant mechanical models. 


\section{ACKNOWLEDGMENTS}

The work presented in this paper is supported jointly by a grant from Shandong Province Natural Science Foundation (No. BS2015NJ016), a grant from Shandong Province Green Building Collaborative Innovation Centre Team Building Foundation (No. LSXT201502), the Doctoral Research Fund of Shandong Jianzhu University (No. XNBS1222), and a grant from Department of Science \& Technology of Shandong Province (No. 2013SJGZ15).

\section{NOMENCLATURE}

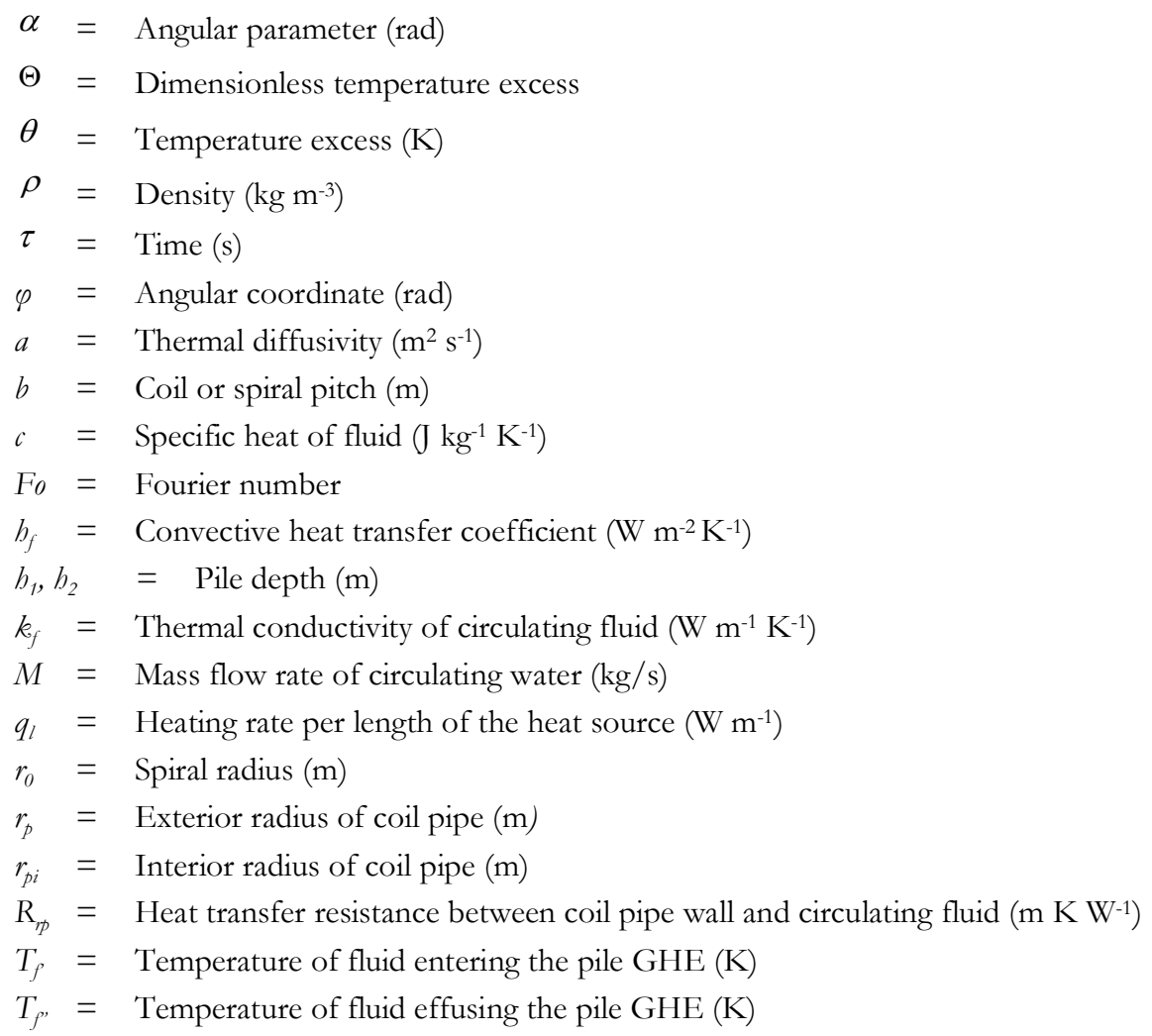

\section{REFERENCES}

Bose J.E., Parker J.D., McQuiston F.C. 1985. Design/ data manual for closed-loop ground coupled heat pump systems. Oklahoma State University for ASHRAE.

Huerta L., Krarti M. 2015. Foundation heat transfer analysis for buildings with thermal piles. Energy Conversion and Management 89: 449-457.

Loveridge F., Powrie W. 2014. 2D thermal resistance of pile heat exchangers. Geothermics 50: 122-135.

Luo J., Zhao H., Gui S., Xiang W., Rohn J., Blum P. 2016. Thermo-economic analysis of four different types of ground heat exchangers in energy piles. Applied Thermal Engineering 108: 11-19.

Mehrizi A., Porkhial S., Bezyan B., Lotfizadeh H. 2016. Energy pile foundation simulation for different configurations of ground source heat exchanger. International Communications in Heat and Mass Transfer 70: 105-114.

Rohsenow W.M. and Hartnet J.P.T. 1983. Handbook of Heat Transfer, McGraw Hill, 2-nd.

Suryatriyastuti M.E., Mroueha H., Burlon S. 2012. Understanding the temperatureinduced mechanical behaviour of energy pile foundations. Renewable Sustainable Energy 16 (2012) 3344-3354.

Xiang Y., Su H., Gou W.S., Zhao Y., Kuang W.J., Liu Z.J., Wu Y. 2015. A new practical numerical model for the energy pile with spiral coils. International Journal of Heat and Mass Transfer 91(2015): 777-784. 